\title{
Review of Self-Reported Data from UK Do-It-Yourself Artificial Pancreas System (DIYAPS) Users to Determine Whether Demographic of Population Affects Use or Outcomes
}

Tim J. Street

Received: October 6, 2020 / Accepted: April 30, 2021 / Published online: May 28, 2021

(C) The Author(s) 2021

\section{ABSTRACT}

Introduction: This study investigates the use of do-it-yourself artificial pancreas systems (DIYAPS) in the UK, and whether self-reported outcomes associated with them are affected by the demographics of the user.

Methods: An online survey was conducted to assess user demographic data and self-reported time-in-range (TIR) outcomes. Statistical analysis was undertaken to review the results and check whether there were major differences in change in TIR across age, gender and duration of diabetes.

Results: Of 253 valid responses to the survey across a wide age range of users, $74.4 \%$ related to adult users and $25.6 \%$ related to under- $16 \mathrm{~s}$. The majority (65.6\%) used AndroidAPS, but there was greater use of Loop (43.1\%) amongst under-16s than amongst adults $(25.9 \%)$. Correspondingly, more under-16s (40.0\%) than adults (24.3\%) used Omnipod Eros. A 17.3\% $( \pm 13.7 \%)$ increase in time in range was reported across all participants, with no significant differences observed between age groups, genders or diabetes duration groups $(p>0.05)$.

Supplementary Information The online version contains supplementary material available at https:// doi.org/10.1007/s13300-021-01071-5.

T. J. Street $(\square)$

Diabettech Ltd, London, UK

e-mail: tim@diabettech.com
Conclusions: The results show that these systems are being used by both genders, and that users cover a wide range of ages and diabetes durations. They also show that improvements in self-reported DIYAPS healthcare outcomes may not be specific to any particular age, gender or duration of diabetes, and the results may provide additional insights into the applicability of the algorithms by demographic. This study may also inform healthcare professionals about the use and effectiveness of DIYAPS solutions.

Keywords: Diabetes; DIYAPS; OpenAPS; Loop; AndroidAPS; WeAreNotWaiting; Do-it-yourself artificial pancreas system; Hybrid closed loop; Open source; Demographics; Automated insulin delivery 


\section{Key Summary Points}

Why carry out the study?

The study was undertaken to provide insight into the users of DIYAPS in the UK and to determine whether there are specific demographics using them.

The study also attempted to determine whether demographics affected time-inrange (TIR) outcomes when using DIYAPS.

\section{What was learned from the study?}

Users of DIYAPS in the UK who responded to the survey had a range of ages and durations of diabetes and were of both genders.

There were clear peaks in use amongst 10to 15 -year-olds and 36- to 40-year-olds, with gender playing no part in the average age or duration of diabetes of the participants.

The DIYAPS used were mostly split between AndroidAPS and Loop systems.

There was no statistically significant difference in self-reported increase in TIR between the participants when split by age, gender or duration of diabetes.

\section{DIGITAL FEATURES}

This article is published with digital features, including a summary slide, to facilitate understanding of the article. To view digital features for this article go to https://doi.org/10.6084/ m9.figshare.14510268.

\section{INTRODUCTION}

While improvements in treatments and technology available to people with diabetes continue to be made, a minority of people achieve clinically recommended targets for $\mathrm{HbA}_{1 \mathrm{c}}$ [1].
Whilst a range of solutions that aim to remove the burden of living with diabetes are now commercially available, they are not universally accessible or affordable to all.

Prior to and alongside this, a community of people with diabetes have built their own algorithms that are designed to work with commercially available pumps and continuous glucose monitoring devices to provide access to similar technology [2]. This is known as the \#WeAreNotWaiting community. Source code for the available systems is available to download for free, but users are required to build the systems themselves and must take personal responsibility for building and maintaining the system and running it at their own risk.

Self-reported outcomes have been published [3-7] that provide a range of data on effectiveness. The common themes reported are:

- Improved time in range (TIR)

- Improved sleep quality

- Reduced glucose variability

- A reduction in time spent thinking about diabetes.

While DIYAPS users are perceived to be a "self-selected group of technical, motivated and highly engaged individuals" [8], a PubMed search revealed little data relating to the demographics of users and whether the outcomes differ by demographic. The Association of British Clinical Diabetologists (ABCD) recently launched a DIYAPS audit [9] to capture some of these data, but it is far from complete.

This study assesses self-reported TIR outcomes amongst that population and identifies whether there is a particular demographic that has obtained a greater increase. It also assesses the demographics of users in the UK and tests the hypothesis that there may be greater representation from a particular age group, gender or diabetes duration group.

\section{METHOD}

An online survey containing 15 questions (see the Supplementary Information: Survey Questions) was published on http://www.diabettech. com for approximately 3 weeks from June 25 th 
to July 14th. The survey was distributed using Twitter, the Facebook groups "Looped UK" (> 2000 members as of July 1st 2020) and "AndroidAPS Users" ( $>4000$ members as of July 1st 2020), and Instagram.

The survey questions covered three main areas:

- User demographics, including age, gender and duration of living with diabetes

- Types of technology used and duration of use

- TIR outcomes observed both before and after the DIYAPS was used.

The survey was an enhanced version of previous surveys undertaken on Diabettech.com $[10,11]$, to include TIR data.

Following the closure of the survey, the data were reviewed and cleansed to remove duplicate data and any data completed by persons from outside the UK. This was done by removing data for which the participant's location was listed as outside the UK. An additional check in which $10 \%$ of the captured IP addresses were sampled to determine whether they came from UK or non-UK sources was also undertaken.

Exploratory analysis of the results set was undertaken. Where it was not possible to interpret the data provided, the responses were removed from the dataset. Any responses that did not include data associated with the area of analysis (e.g. age, system type or TIR) were excluded from that analysis.

The data were then split according to the demographics that were being assessed:

- Age

- Gender

- Duration of living with diabetes.

Where the data was related to time, it was grouped into periods of 10 years to ensure meaningful sample sizes.

The arithmetic mean, SD, Mann-Whitney $U$ test, two-tailed heteroscedastic $t$ test, and analysis of variance (ANOVA) [12] were used to perform the statistical analysis, which was undertaken using GraphPad Prism for Windows. An alpha level of 0.05 was used for all analyses. This questionnaire was categorised as "Not Research", so an Ethics Committee approval was deemed not to be required. Survey participation was entirely voluntary, therefore completion was deemed to be consent to participate.

\section{RESULTS}

Overall, 296 participants responded to the survey, of whom 253 were validated as being from the UK by checking IP addresses and removing duplicated entries. Of the total, $43.1 \%$ were female, with a median age of 35 years (range 1-70 years). The median duration of diabetes was 19.5 (SD 14.1) years (range 0-61 years). Various types of DIYAPS (AndroidAPS 65.6\%, Loop $30.4 \%$ and OpenAPS 3.2\%) were being used.

\section{Demographics of DIYAPS Use}

There were $31.1 \%$ more male than female respondents to the survey, and the distribution by age was bimodal, with peaks at ages 40-45 and $10-15$ years. $25.6 \%$ of users were under 16 . The age and gender distribution of the participants is shown in Fig. 1.

The Mann-Whitney $U$ test was used to determine whether gender influenced participant age or duration of diabetes. The means and standard deviations can be found in Table 1 .

There was no significant difference in age (Mann-Whitney $U=7109, n_{1}=109, n_{2}=141$, $p=0.3104$ two-tailed) or duration of diabetes (Mann-Whitney $U=7096, n_{1}=109, n_{2}=142$, $p=0.2592$ two-tailed) between the genders. The median age was 35.0 years for females and 37.0 years for males. The median duration of diabetes was 18 years for females and 21 years for males.

The average age of Loop users (28.5 years) was substantially lower than those of AndroidAPS (35.8 years) and OpenAPS (33 years) users as shown in Table 2.

Adult Loop users were more likely to use Omnipod Eros pumps (72.9\%) than older Medtronic pumps (27.1\%), while adult AndroidAPS users were distributed more evenly across pump types, with the DanaRS pump (34.6\%) being the most popular. A greater proportion of adult male users $(80.0 \%)$ of Loop used Omnipod Eros 


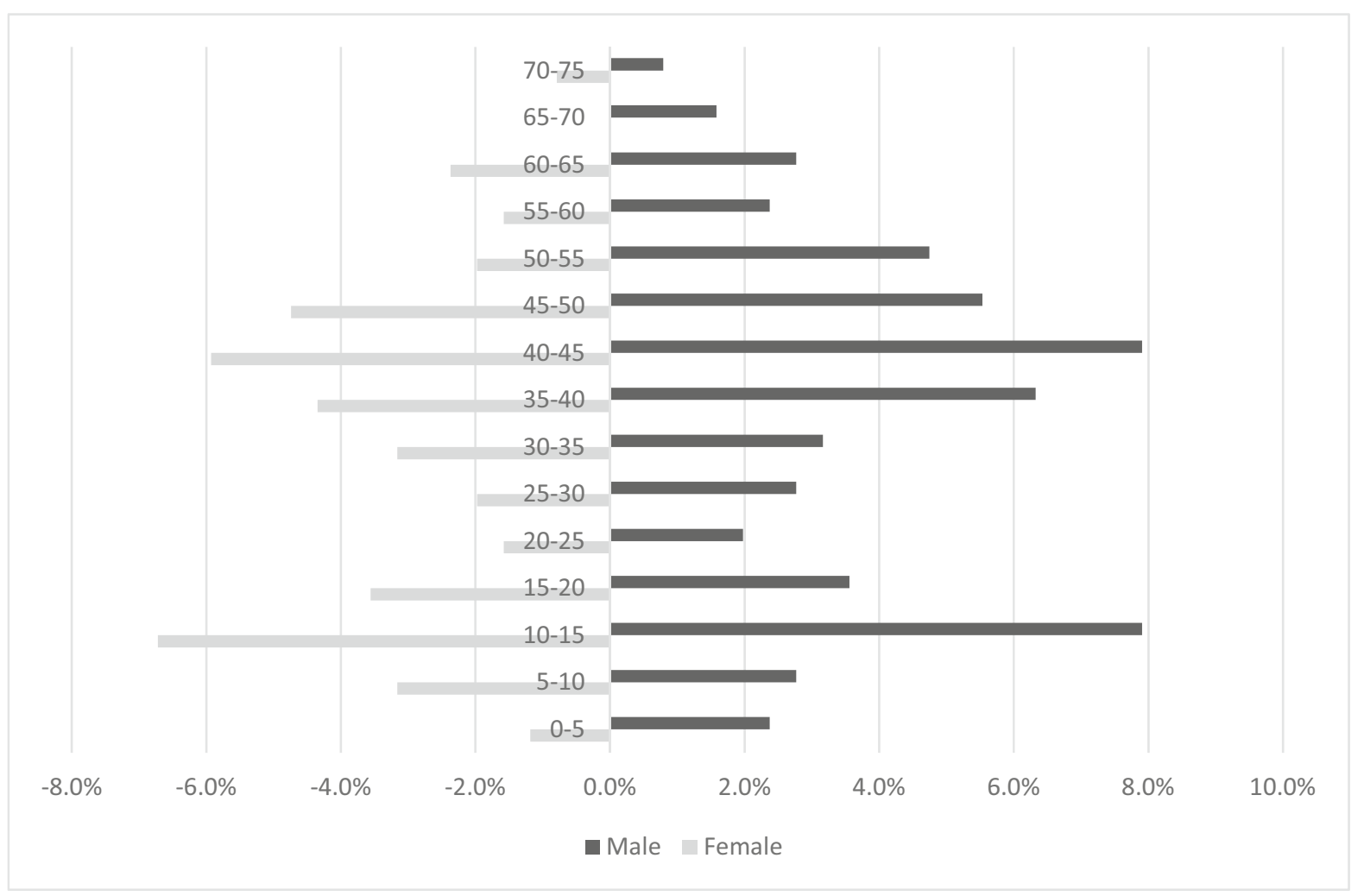

Fig. 1 Age and gender distribution of the survey participants

than female users (65.2\%). Loop was more popular amongst adult female users (29.5\%) than with adult male users (23.4\%), although AndroidAPS was the most popular system in both cases $($ male $=71.0 \%$; female $=69.2 \%)$. These details can be found in Table 3 .

Amongst the under-16s, there were more AndroidAPS users (53.8\%) than Loop users (43.1\%); however, compared to the adult population $(25.9 \%)$, this age group made greater use of Loop. Most of the under-16 AndroidAPS users were using the Roche Accuchek Combo pump (48.6\%), in contrast to the under-16 Loop users, most of whom used the Omnipod Eros (92.3\%). Amongst the under-16s, the most popular pump choice was the Omnipod Eros (40.0\%). These details can be found in Table 4 .

\section{Outcomes from DIYAPS Use}

$47.4 \%$ of the survey participants provided TIR data for the 3 months prior to the first use of the
DIYAPS, as well as the TIR for the previous 3 months whilst using the system. The average duration of use of the DIYAPS by these users was 10.3 months. All participants saw a mean increase in TIR of 17.3 (SD 13.7) percentage points.

Welch's $t$ test was used to examine whether gender influenced change in TIR. See Table 5 for means and standard deviations.

Women $(M=16.4, \mathrm{SD}=13.4)$ presented a lower increase in TIR than men $(M=18.1$, $\mathrm{SD}=14.0)$; however, Welch's $t$ test deemed that the difference between the TIR increases of men and women was not statistically significant: $t(119)=0.67, p=0.5013$.

A two-way analysis of variance (ANOVA) between age groups was used to examine the effect of age on change in TIR. There were 7 age groups: $0-10,11-20,21-30,31-40,41-50$, $51-60$, and $>60$ years. The dependent variable was the change in TIR. See Table 6 for means and standard deviations. 
Table 1 Survey participant demographics and DIYAPS use characteristics

\begin{tabular}{|c|c|c|c|c|c|c|c|}
\hline & \multirow[t]{2}{*}{$n$} & \multirow{2}{*}{$\begin{array}{l}\text { Mean age, years } \\
\text { (SD) }\end{array}$} & \multirow{2}{*}{$\begin{array}{l}\text { Mean duration of diabetes, } \\
\text { years (SD) }\end{array}$} & \multicolumn{4}{|l|}{ DIYAPS } \\
\hline & & & & AndroidAPS & Loop & OpenAPS & $\overline{\text { Unknown }}$ \\
\hline All & 253 & $33.5(17.8)$ & $20.3(14.1)$ & 166 & 76 & 8 & 2 \\
\hline Female & 109 & $32.2(17.6)$ & $19.3(13.9)$ & 69 & 38 & 2 & 0 \\
\hline Male & 143 & $34.5(17.1)$ & $21.2(14.4)$ & 97 & 38 & 6 & 2 \\
\hline $\begin{array}{l}\text { Rather not } \\
\text { say }^{*}\end{array}$ & 1 & * & $*$ & $*$ & * & $*$ & * \\
\hline
\end{tabular}

*Data were obfuscated to maintain anonymity and were not included in the total for all

Table 2 Mean age of participants split by DIYAPS used

\begin{tabular}{lrllll}
\hline & \multicolumn{1}{c}{$\boldsymbol{n}$} & Mean (years) & Std. deviation (years) & Lower 95\% CI of mean & Upper 95\% CI of mean \\
\hline AndroidAPS & 166 & 35.8 & 18.2 & 33.0 & 38.6 \\
Loop & 77 & 28.5 & 16.3 & 24.8 & 32.2 \\
OpenAPS & 8 & 33.3 & 18.8 & 17.5 & 49.0 \\
\hline
\end{tabular}

The two-way Welch's ANOVA of the 7 age groups revealed no significant effect of age on change in TIR at the $p<0.05$ level $[F(6.0$, 33.6) $=0.881, p=0.5192]$.

Post hoc comparisons using Dunnett's T3 multiple comparisons procedure showed no significant differences in pairwise comparisons.

A two-way ANOVA between diabetes duration groups was used to examine the effect of duration of diabetes on change in TIR. There were 5 diabetes duration groups: 0-10, 11-20, $21-30,31-40$, and $>41$ years. See Table 7 for means and standard deviations.

The two-way Welch's ANOVA of the 5 diabetes duration groups revealed no significant effect of duration of diabetes on change in TIR at the $p<0.05$ level $[F(4,38.73)=1.748$, $p=0.159]$. Post hoc comparisons using Dunnett's T3 multiple comparisons procedure also showed no significant differences in pairwise comparisons.

\section{DISCUSSION}

This survey adds to the dataset of TIR outcomes for users of all DIYAPS across gender, age and duration of diabetes. To the best of the author's knowledge, it is also the largest review of users of these systems in the UK to see a significant increase in self-reported TIR (17.3\%) for DIYAPS users.

\section{User Age, Gender and Duration of Diabetes}

The distribution in age can be seen to be bimodal across male and female participants, with the same modality reflected in both genders. The first peak corresponds to children aged 5-10 years, whilst the second corresponds to adults aged 40-45. The first peak is likely to be driven by parents attempting to help their children to improve their sleep and glycaemia [7]. The second peak is likely to correspond to an age where improvements in long-term outcomes and overall glycaemia become more important to adults [7]. There was no significant difference in mean age between male and female users.

The mean age of the users who responded to the survey was 33.4 years, but mean age depended on the system used, with Loop users having a substantially lower mean age than 
Table 3 System and pump use by adult survey participant gender

\begin{tabular}{lrrlll}
\hline & \multicolumn{2}{l}{ DIYAPS } & & & \\
\cline { 2 - 5 } & \multicolumn{1}{c}{$\boldsymbol{n}$} & AndroidAPS & Loop & OpenAPS & Unknown \\
\hline Female & 78 & 54 & 23 & 1 & \\
DanaRS & 20 & 20 & & 1 & \\
Medtronic 640G & 2 & 1 & 8 & \\
Medtronic Loopable Pump (Veo) & 10 & 2 & 15 & \\
Omnipod Eros & 18 & 3 & & \\
Roche Accuchek Insight & 11 & 11 & & \\
Roche Accuchek Combo & 17 & 17 & 25 & 5 \\
Male & 107 & 76 & & \\
DanaR & 1 & 1 & & \\
DanaRS & 25 & 25 & 5 & 5 \\
Medtronic Loopable Pump (Veo) & 13 & 3 & 20 & \\
Omnipod Eros & 27 & 6 & & \\
Roche Accuchek Insight & 23 & 23 & 18 & \\
Roche Accuchek Combo & 18 & 18 & & \\
\hline
\end{tabular}

AndroidAPS users. Compared to previous surveys, a similar proportion of the population reported using AndroidAPS (65.3\%), but Loop $(30.7 \%)$ saw an increase in use compared to the previous year [10]. More than one-quarter of all users were under 16 , and a majority of those used AndroidAPS (53.8\%) rather than Loop (43.1\%) or OpenAPS (3.1\%).

The survey did not ask why a specific system or pump was chosen. However, amongst under16 pump users, it is likely that the choice of pump was heavily influenced by their caregivers. The high prevalence of Loop users among the under-16s compared to the general survey population (43.1\% compared to $30.4 \%$ ) and the significant use of Omnipod Eros amongst the under-16 users (85.7\%) suggest that the selection of Omnipod drives the selection of Loop (the only DIYAPS that supported Omnipod at the time of the survey) by providing both an "in-warranty" pump option and the only tubeless pump option that is currently available to use with a DIYAPS.
Across both male and female participants, AndroidAPS was the most popular system choice. Reviewing the data in Tables 3 and 4, it can be seen that a wide range of pumps are in use on AndroidAPS, which suggests that those who started therapy using AndroidAPS may have done so with a pump that they were already using. Users who specified that they used a DanaRS pump, which has only become available relatively recently, may have changed pump in order to use AndroidAPS. Reasons for using the DanaRS are not given, but it is one of the few pumps on the market that remains within warranty when used with AndroidAPS, and it is hard to discount this as a driving factor.

Amongst those participating, 30.4\% had lived with diabetes for fewer than 10 years, adding to the suggestion that-as the OPEN Project found-more recently diagnosed people may be taking long-term outcomes into account when choosing to use a DIYAPS [7]. Another potential factor is the technical challenge that may be presented to those who have a longer duration of diabetes; however, the technical 
Table 4 System and pump use by juvenile survey participant gender

\begin{tabular}{|c|c|c|c|c|}
\hline & \multicolumn{4}{|c|}{ DIYAPS } \\
\hline & $n$ & AndroidAPS & Loop & OpenAPS \\
\hline Female & 31 & 15 & 15 & 1 \\
\hline DanaRS & 2 & 2 & & \\
\hline $\begin{array}{l}\text { Medtronic } \\
640 \mathrm{G}\end{array}$ & 1 & 1 & & \\
\hline $\begin{array}{l}\text { Medtronic } \\
\text { Loopable } \\
\text { Pump (Veo) }\end{array}$ & 4 & & 3 & 1 \\
\hline Omnipod Eros & 14 & 1 & 12 & \\
\hline $\begin{array}{l}\text { Roche } \\
\text { Accuchek } \\
\text { Insight }\end{array}$ & 5 & 5 & & \\
\hline $\begin{array}{l}\text { Roche } \\
\text { Accuchek } \\
\text { Combo }\end{array}$ & 6 & 6 & & \\
\hline Male & 34 & 20 & 13 & 1 \\
\hline DanaRS & 7 & 7 & & \\
\hline $\begin{array}{l}\text { Medtronic } \\
\text { Loopable } \\
\text { Pump (Veo) }\end{array}$ & 2 & & 1 & 1 \\
\hline Omnipod Eros & 12 & & 12 & \\
\hline $\begin{array}{l}\text { Roche } \\
\text { Accuchek } \\
\text { Insight }\end{array}$ & 2 & 2 & & \\
\hline $\begin{array}{l}\text { Roche } \\
\text { Accuchek } \\
\text { Combo }\end{array}$ & 11 & 11 & & \\
\hline
\end{tabular}

ability of users to build one of these systems was not assessed in the survey. There was no significant difference in mean duration of diabetes between male and female users.

\section{Improvements in Outcomes}

As a secondary dataset, the changes in TIR provide some positive feedback. Respondents reported an increase in mean TIR of $17.3 \%$ (equating to an overall increase of $27.1 \%$ ). This corresponds to an estimated reduction in $\mathrm{HbA}_{1 \mathrm{c}}$ of approximately $1.0-1.6 \%(11-15 \mathrm{mmol} / \mathrm{mol})$ according to the recent consensus in TIR publication [13].

Across all users, whether stratified by age, gender or duration of diabetes, there were no statistically significant differences in mean change in TIR. Although the survey was not powered to determine it, this suggests that the two DIYAPS algorithms in use are not sensitive to age, gender or length of time that a user has lived with diabetes.

\section{Strengths and Limitations}

This study is the first to investigate differences in outcomes from using DIYAPS based on the demographics of respondents. It adds to the cohort of studies reporting self-reported outcomes of people with diabetes in general. It surveyed a large proportion of the DIYAPS users in the UK, with 253 respondents from a total population estimated at $N=750$ in 2020, based on the number of reported users of AndroidAPS and the number of Rileylinks shipped to unique addresses in the UK.

A key limitation of the study is that it is selfreported and outcomes were not corroborated by clinical records. This is often put forward as

Table 5 Self-reported mean change in TIR of participants by gender

\begin{tabular}{|c|c|c|c|c|}
\hline & $N$ & Mean TIR pre-DIYAPS use \% & Mean TIR post-DIYAPS use \% & Mean change in TIR \% \\
\hline All & 119 & $63.9(17.2)$ & $81.3(11.1)$ & $17.3(13.7)$ \\
\hline Female & 51 & $64.3(15.9)$ & $80.6(9.2)$ & $16.4(13.4)$ \\
\hline Male & 68 & $63.7(18.2)$ & $81.7(12.4)$ & $18.1(14.0)$ \\
\hline
\end{tabular}


Table 6 Self-reported mean change in TIR of participants by age group

\begin{tabular}{lcllcl}
\hline $\begin{array}{l}\text { Age } \\
\text { (years) }\end{array}$ & $\boldsymbol{N}$ & $\begin{array}{l}\text { Mean } \\
\text { \% }\end{array}$ & $\begin{array}{l}\text { Std. } \\
\text { deviation } \\
\text { \% }\end{array}$ & $\begin{array}{l}\text { Lower } \\
\mathbf{9 5 \%} \mathbf{C I}\end{array}$ & $\begin{array}{l}\text { Upper } \\
\mathbf{9 5 \%} \mathbf{C I}\end{array}$ \\
\hline $0-10$ & 12 & 13.1 & 11.4 & 5.9 & 20.3 \\
$11-20$ & 31 & 17.4 & 12.2 & 12.9 & 21.9 \\
$21-30$ & 11 & 30.5 & 25.2 & 13.5 & 47.4 \\
$31-40$ & 24 & 17.5 & 11.5 & 12.6 & 22.4 \\
$41-50$ & 22 & 14.8 & 9.6 & 10.5 & 18.99 \\
$51-60$ & 13 & 14.3 & 13.7 & 6.1 & 22.6 \\
$>60$ & 6 & 16.8 & 8.4 & 8.0 & 25.7 \\
\hline
\end{tabular}

Table 7 Self-reported mean change in TIR of participants by diabetes duration group

\begin{tabular}{llllll}
\hline $\begin{array}{l}\text { Diabetes } \\
\text { duration } \\
\text { (years) }\end{array}$ & $n$ & $\begin{array}{l}\text { Mean } \\
\%\end{array}$ & $\begin{array}{l}\text { Std. } \\
\text { deviation } \\
\%\end{array}$ & $\begin{array}{l}\text { Lower } \\
\mathbf{9 5 \%} \\
\text { CI }\end{array}$ & $\begin{array}{l}\text { Upper } \\
\mathbf{9 5 \%} \\
\text { CI }\end{array}$ \\
\hline $0-10$ & 46 & 15.99 & 14.69 & 11.63 & 20.35 \\
$11-20$ & 19 & 21.94 & 18.06 & 13.24 & 30.65 \\
$21-30$ & 25 & 18.6 & 10.41 & 14.3 & 22.9 \\
$31-40$ & 19 & 13.29 & 6.528 & 10.14 & 16.44 \\
$>41$ & 10 & 19.5 & 16.06 & 8.013 & 30.99 \\
\hline
\end{tabular}

an argument for the presentation of inaccurate data, but a Norwegian study found that realworld patient-reported data are aligned with data from patient registries reported by health care professionals [14]. DIYAPS users constitute a specific group who are highly motivated and engaged and thus may be more motivated to participate in a survey such as this. As a selfselecting participant group, it is possible that those with less preferential outcomes may not have participated, generating a participant bias in the reported outcomes. Further investigation using controlled trials would be required to verify that the data published here are reflective of normal use.
The survey questions did not cover the various customisations and variations that are possible across the systems, so the impact that these may have had is unclear. It also did not review the amount of manual input provided by users to achieve outcomes. Another area that was not investigated in the survey was time below range (TBR), as the difference in change in TIR between participating demographic groups was the secondary investigatory outcome of the study. As this is a key concern relating to DIYAPS for healthcare professionals, it is recommended that future studies include TBR. The analysis also did not look at whether a specific system works more effectively for one specific group than another; only whether it was no different between groups. The survey was openly accessible on the Internet and it is therefore possible that respondents were not from the UK, even when they may have stated that they were. It is also possible that responses were faked or duplicated, although some validation of the responses based on IP address origins and data deduplication was undertaken. An online survey such as this may also lead to bias in the gender of the respondents.

Additionally, this study was undertaken by a member of the diabetes community, which could be considered a strength of the study but could also mean that it has the potential for bias.

\section{CONCLUSIONS}

There is greater self-reported use of DIYAPSs amongst survey participants aged 10-15 and $35-40$ years in the UK and amongst men than women; however, people of all ages, with all durations of diabetes and genders have found DIYAPS to be effective and have chosen to use them over regular therapies. The use of DIYAPS systems resulted in increased TIR amongst participants in this self-reported survey.

The algorithms used by the various systems are shown to result in statistically significant increases in mean TIR, effective regardless of age, gender or duration of diabetes. The WeAreNotWaiting movement has created solutions that are both effective and usable by a 
wide range of people, demonstrating how patients are able to drive innovation in diabetes care.

\section{ACKNOWLEDGEMENTS}

The author acknowledges the contribution of members of the WeAreNotWaiting community and UK Diabetes Online Community that participated in the survey. The author would like to thank Dr Sufyan Hussain for helpful comments and feedback on the manuscript.

Funding. No funding or sponsorship was received for this study or publication of this article.

Authorship. All named authors meet the International Committee of Medical Journal Editors (ICMJE) criteria for authorship for this article, take responsibility for the integrity of the work as a whole, and have given their approval for this version to be published.

Disclosures. Tim J Street: Guest blog for DiabetesMine plus expenses covered to speak at DData 2019; Co-organiser of "Rise of the Machine" events; Expenses covered to speak at EASD Postgraduate Course on Diabetes and Technology 2018; Expenses covered by Diabetes UK Cymru to speak at their "Type 1 and Tech" conference 2018 and 2019; Expenses covered by Cambridge University for attending a focus group on their artificial pancreas; Sponsored by Abbott Diabetes Care to attend DX 2019 in Lisbon on June 21st 2019; Paid speaker for ABCD meeting in 2019; NightScout Foundation covered ATTD2020 registration fee.

Compliance with Ethics Guidelines. This questionnaire was categorised as "Not Research", so an Ethics Committee approval was deemed not to be required. Survey participation was entirely voluntary, therefore completion was deemed to be consent to participate.

Data Availability. The datasets generated during and/or analysed during the current study are available from the corresponding author on reasonable request.

Open Access. This article is licensed under a Creative Commons Attribution-NonCommercial 4.0 International License, which permits any non-commercial use, sharing, adaptation, distribution and reproduction in any medium or format, as long as you give appropriate credit to the original author(s) and the source, provide a link to the Creative Commons licence, and indicate if changes were made. The images or other third party material in this article are included in the article's Creative Commons licence, unless indicated otherwise in a credit line to the material. If material is not included in the article's Creative Commons licence and your intended use is not permitted by statutory regulation or exceeds the permitted use, you will need to obtain permission directly from the copyright holder. To view a copy of this licence, visit http://creativecommons.org/licenses/by$\mathrm{nc} / 4.0 /$.

\section{REFERENCES}

1. NHS Digital. Care Processes and Treatment Targets, January to December 2019. 11 June 2020. Retrieved from NHS Digital: https://digital.nhs.uk/data-andinformation/publications/statistical/nationaldiabetes-audit/national-diabetes-audit-quarterlyreport-january-to-december-2019. Accessed 27 Aug, 4 Sep 2020.

2. Kesavadev J, Srinivasan S, Saboo B, et al. The do-ityourself artificial pancreas: a comprehensive review. Diabetes Ther. 2020;11:1217-35. https://doi.org/10. 1007/s13300-020-00823-Z.

3. Hng TM, Burren D. Appearance of do-it-yourself closed-loop systems to manage type 1 diabetes. Intern Med J. 2018;48:1400-4. https://doi.org/10. 1111/imj.14105.

4. Lee JM, Newman MW, Gebremariam A, Choi P, Lewis D, Nordgren W, et al. Real-world use and selfreported health outcomes of a patient-designed doit-yourself mobile technology system for diabetes: lessons for mobile health. Diabetes Technol Ther. 2017;19:209-19. https://doi.org/10.1089/dia.2016. 0312. 
5. Melmer A, Züger T, Lewis DM, Leibrand S, Stettler C, Laimer M. Glycemic control in individuals with type 1 diabetes using an open source artificial pancreas system (OpenAPS). Diabetes Obes Metab. 2019. https://doi.org/10.1111/dom.13810.

6. Petruzelkova L, Soupal J, Plasova V, Jiranova P, Neuman V, Plachy L, et al. Excellent glycemic control maintained by open-source hybrid closedloop AndroidAPS during and after sustained physical activity. Diabetes Technol Ther. 2018;20: 744-50. https://doi.org/10.1089/dia.2018.0214.

7. Braune K, O’Donnell S, Cleal B, Lewis D, Tappe A, Willaing I, et al. Real-world use of do-it-yourself artificial pancreas systems in children and adolescents with type 1 diabetes: online survey and analysis of self-reported clinical outcomes. JMIR MHealth UHealth. 2019;7:e14087. https://doi.org/ $10.2196 / 14087$.

8. Jennings P, Hussain S. Do-it-yourself artificial pancreas systems: a review of the emerging evidence and insights for healthcare professionals. J Diabetes Sci Technol. 2019. https://doi.org/10.1177/ 1932296819894296.

9. Association of British Clinical Diabetologists. ABCD do-it-yourself artificial pancreas system (DIY APS) audit. July 2020. Retrieved from ABCD: https:// abcd.care/DIYAPS\#: :text=About $\% 20$ the $\%$ 20ABCD\%20nationwide\%20DIY,may\%20help\% 20inform\%20future\%20practice. Accessed 27 Aug 2020.

10. Street $\mathrm{T}$ (2018). \#DIYAPS use in the UK-a snapshot. Retrieved from Diabettech: https://www. diabettech.com/artificial-pancreas/diyaps-use-inthe-uk-a-snapshot/. Accessed 27 Aug 2020.

11. Street T (2019) May 2019 snapshot of UK DIY APS users. Retrieved from Diabettech: https://www. diabettech.com/artificial-pancreas/may-2019. snapshot-of-uk-diy-aps-users/. Accessed 27 Aug 2020.

12. Blanca MJ, Alarcón R, Arnau J, Bono R, Bendayan R. Non-normal data: is ANOVA still a valid option? Psicothema. 2017;29(4):552-7. https://doi.org/10. 7334/psicothema2016.383.

13. Battelino $\mathrm{T}$, Danne $\mathrm{T}$, Bergenstal RM, Amiel SA, Beck R, Biester T, et al. Clinical targets for continuous glucose monitoring data interpretation: recommendations from the international consensus on time in range. Diabetes Care. 2019;42:1593-603. https://doi.org/10.2337/dci19-0028.

14. Løvaas KF, Cooper JG, Sandberg S, Røraas T, Thue G. Feasibility of using self-reported patient data in a national diabetes register. BMC Health Serv Res. 2015;15(15):553. https://doi.org/10.1186/s12913015-1226-0. 